\title{
HERPES SIMPLEX VIRUS: A REVIEW OF CURRENT PRACTICE OF TREATMENT AND SUPPRESSION AT ST. JAMES'S HOSPIAL DUBLIN VERSUS 2017 EUROPEAN GUIDELINES FOR THE MANAGEMENT OF GENITAL HERPES
}

\author{
Joyce Donnelly ${ }^{1}$, Niamh Lynn², Miriam Moriarty ${ }^{1}$, Gail Melanophy루, Fiona Mulcahy². \\ 1. Pharmacy Department, St James's Hospital
}

Introduction

2. Department of Genito-Urinary Medicine and infectious diseases (GUIDe), St. James's Hospital

Genital Herpes is a common sexually transmitted infection. ${ }^{1}$ In 2017 , there were 1,564 cases reported in Ireland. ${ }^{2}$ Early diagnosis and prompt initiation of therapy may reduce the duration of illness and avoid complications such as urinary retention and severe systemic illness.

Most early studies of Herpes Simplex Virus (HSV) suppressive therapy looked at patients with a recurrence rate of $\geq 6$ recurrences per annum. ${ }^{1}$ Recent studies have included patient with milder disease which would indicate that patients with all severities of disease could benefit from suppressive treatment; if started, the decision to continue should be reviewed annually. European Guidelines for the management of Genital Herpes recommend HSV typing with first dose genital herpes to guide treatment and counselling as the frequency of recurrence is greater for HSV-2; HSV-1 is an uncommon cause of recurrent genital disease.

The most experience of suppressive therapy is with $800 \mathrm{mg}$ aciclovir in 2-4 divided doses. Valaciclovir $250 \mathrm{mg}$ twice a day has been shown to be as effective, and there is some debate whether $500 \mathrm{mg}$ once a day is as effective. ${ }^{1}$ For patients experiencing $\leq 10$ recurrences/annum $500 \mathrm{mg}$ once a day is thought to be adequate but those who have $\geq 10$ recurrences/annum, $250 \mathrm{mg}$ twice a day or $1 \mathrm{~g}$ once a day is required.

There is some evidence to support increased antiviral dosing in HIV-positive patients although suppression seems to be less effective in those with HIV. Valaciclovir is most effective when given 500mg twice a day instead of $1 \mathrm{~g}$ once a day. ${ }^{1}$ Herpes simplex infections activate HIV replication and may contribute to onward HIV transmission to partners. Suppression of HSV-2 virus with valaciclovir in HIV positive women not on ARVs has been shown to reduce HIV viral shedding. ${ }^{1}$ The aim of this audit is to review current prescribing practices of HSV suppressive therapy in the Genitourinary Medicines and Infectious Diseases Department (GUIDe), St. James's Hospital, against

recommendations from European Guidelines.

\section{Objectives}

> To assess adherence to recently published European treatment guidelines on the management of Genital Herpes in GUIDe clinic, St. James's Hospital

$>$ To quantify and describe the breakdown of patients receiving HSV treatment in terms of HSV typing, choice of treatment, dosing, episodic or suppressive, follow up, and patient counselling.

$>$ To outline any findings and recommendations based on current guidelines.

\section{Methodology}

A retrospective review, using the electronic prescribing record of 40 patients seen between $1^{\text {st }}$ January- $28^{\text {th }}$ February 2018 who were prescribed HSV treatment was undertaken to calculate:

1. Percentage of HSV typing performed and the ratio of HSV-1 to HSV-2

Number of patients prescribed episodic versus suppressive antivirals

For those on suppression:

a. \% HSV-1 versus HSV-2

b. Rates of annual review

c. \% who had $\geq 6$ episodes per annum prior to suppression

d. Comparison of antiviral dosing in HIV versus STI patients

4. $\%$ of patients who received counselling from a Health advisor

\section{Results}

1. 40 patients identified: 30 HIV Negative/10 HIV Positive - see Graph 1

a) HSV typing performed in $82.5 \%$ ( $n=33)$ - See Graph 2

Of those with HSV-1

* $23 \%(n=3)$ on suppression

$77 \%(n=10)$ on episodic treatment

b) $65 \%(n=26)$ on suppressive therapy

2. HIV-positive patients: $80 \%(\mathrm{n}=8)$ on suppressive therapy; $50 \%(\mathrm{n}=5)$ with documented HSV typing, and $30 \%(n=3)$ without annual review who were eligible

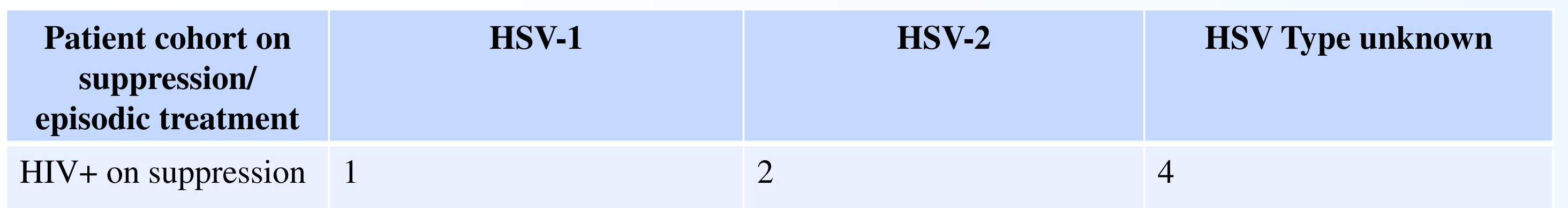

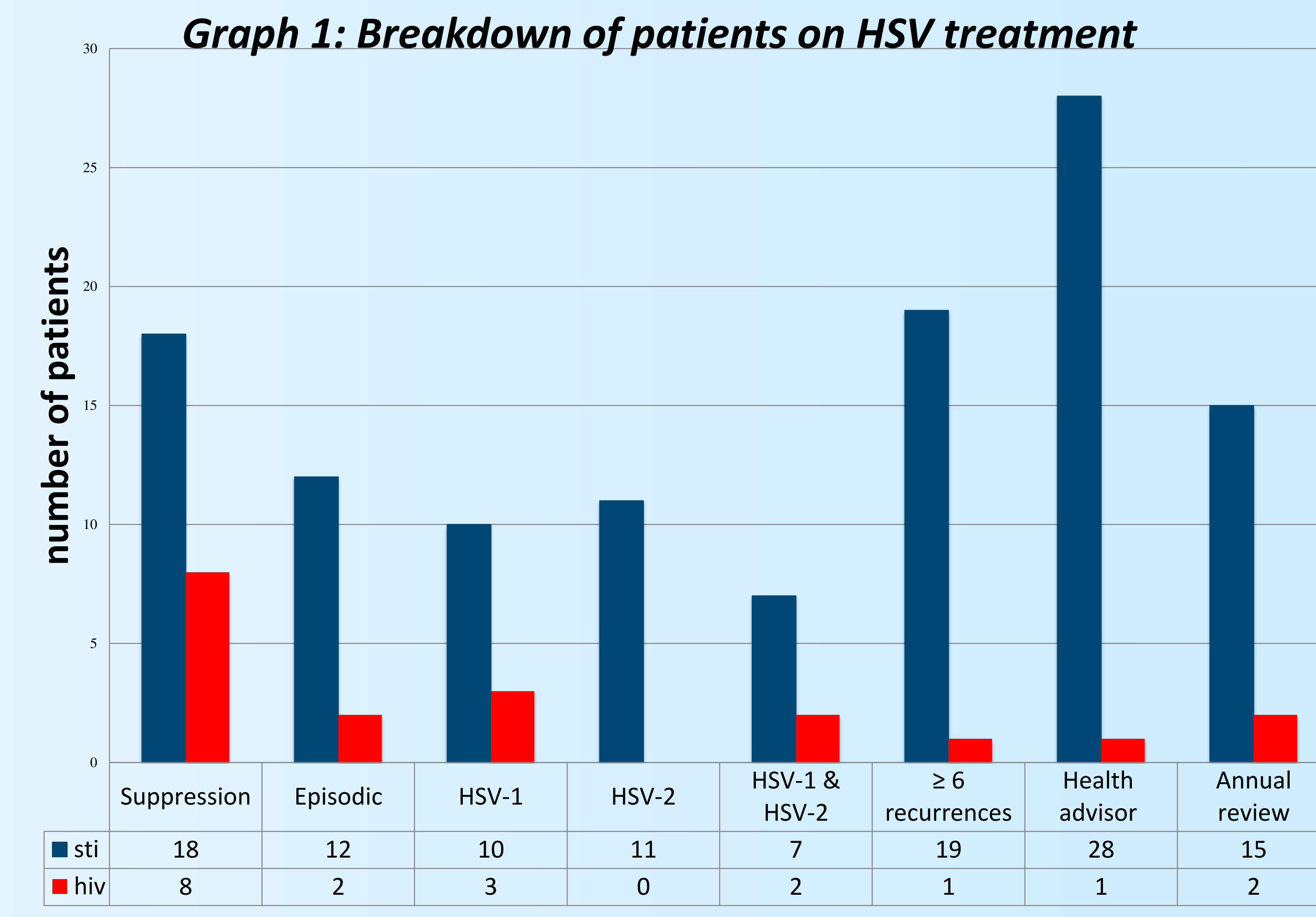
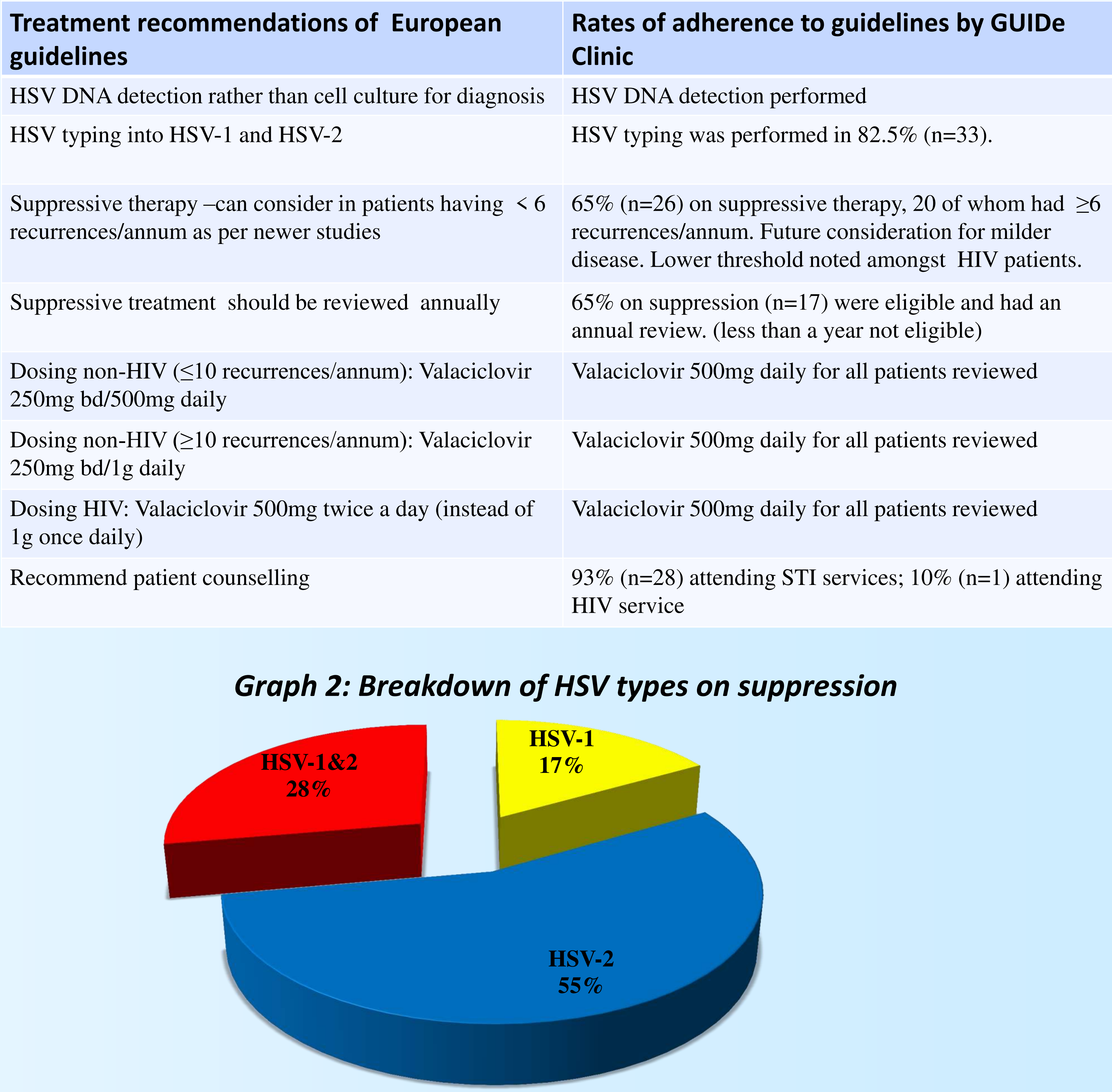

As expected, patients with HSV-1 infection are smallest cohort on suppression. In comparison $77 \%(n=10)$ HSV-1 are on episodic treatment.

\section{Discussion and Recommendations}

Currently valaciclovir $500 \mathrm{mg}$ once daily is the standard dose and treatment prescribed for HSV suppression in all 26 patients regardless of HIV status. This audit has highlighted the need to review antiviral suppressive dosing for HSV in our STI and HIV cohort.

The data demonstrates good compliance with European guidelines in our STI cohort with $87.5 \%$ having HSV typing performed and $93 \%$ receiving counselling. There is room for improvement in documentation of HSV typing, dosing of suppression, referral for counselling, and annual review of suppressive therapy for patients attending HIV services.

The following needs to be addressed at departmental level to discuss

- Review of antiviral dosing in HIV positive patients attending the service

- Review of management of those with milder disease and $<6$ episodes per year with a consideration to offering suppressive therapy

- Review how many patients are having breakthrough episodes while on suppression

- Increase the \% of those who had their HSV sub-typed, especially in the HIV+ population

- Highlight the need for all patients to receive education from a health advisor

\section{References}

1. 2017 European guidelines for the management of genital herpes. Accessed on 01.03.2018 from

2. Health Protection surveillance centre. Accessed on 01.03.2018 from 